\title{
Computerized assessment of social approach behavior in mouse
}

\author{
Damon T. Page ${ }^{1,2 * t}$, Orsolya J. Kuti ${ }^{1,2+}$ and Mriganka Sur ${ }^{1,2}$ \\ 1 Department of Brain and Cognitive Sciences, Massachusetts Institute of Technology, Cambridge, MA, USA \\ 2 Picower Institute for Learning and Memory, Massachusetts Institute of Technology, Cambridge, MA, USA
}

Edited by:

Andrew Holmes, National Institute on Alcohol Abuse and Alcoholism, USA

\section{Reviewed by:}

Tsuyoshi Miyakawa, Kyoto University Japan; Fujita Health University, Japan Craig M. Powell, The University of Texas Southwestern Medical Centre, USA

\section{*Correspondence:}

Damon T. Page, Picower Institute for Learning and Memory, Massachusetts Institute of Technology, 43 Vassar

Street, 46-6227, Cambridge, MA

02139, USA.

e-mail:paged@mit.edu

${ }^{\dagger}$ These authors contributed equally to

this work.
Altered sociability is a core feature of a variety of human neurological disorders, including autism. Social behaviors may be tested in animal models, such as mice, to study the biological basis of sociability and how this is altered in neurodevelopmental disorders. A quantifiable social behavior frequently used to assess sociability in the mouse is the tendency to approach and interact with an unfamiliar mouse. Here we present a novel computer-assisted method for scoring social approach behavior in mice using a three-chambered apparatus and freely available software. We find consistent results between data scored using the computer-assisted method and a human observer, making computerized assessment a reliable, low cost, high-throughput method for testing sociability.

Keywords: social approach, behavior, mouse, autism, genetics

\section{INTRODUCTION}

In the study of social behavior and neuropsychiatric disease, the mouse has become an increasingly popular tool for understanding how brain mechanisms influence behavioral phenotypes, and the number of transgenic mouse models of human disorders available to researchers has risen sharply (Takao et al., 2007). By studying the contribution of individual genes, or the interaction between genes and environment to behavior in animal models, it is possible to uncover the mechanisms by which behavior is altered in human neuropsychiatric disease (Tecott, 2003). Complex behaviors such as aggression, social preference, social memory, maternal care, anxiety, juvenile play and same- and opposite-sex interactions have been studied in mice (Pomerantz et al., 1983; Mondragon et al., 1987; Laviola et al., 1994; Griebel et al., 2000; Blanchard et al., 2001; Ferguson et al., 2001; Long et al., 2004; Rodriguiz et al., 2004; Moretti et al., 2005; O'Tuathaigh et al., 2007; Wersinger et al., 2007), giving insight into the neurobiology of social behavior. In studying mouse models of disorders such as schizophrenia, autism, or William's syndrome, social behaviors are particularly important for testing face validity of a model (Crawley, 2004) and provide an opportunity to assess the efficacy of therapeutics.

A commonly assayed social behavior involves quantifying the tendency for male and female mice to investigate an unfamiliar mouse through mutual sniffing, grooming, or following (Wills et al., 1983; Blanchard and Blanchard, 2003). This social interaction can be measured by observing two mice interacting freely in an open field (Cutler, 1991; Shi et al., 2003), however, in the laboratory this approach can make it difficult to determine which mouse initiates social contact, and may be confounded by undesired interactions such as aggression or mounting (Winslow and Camacho,
1995). To isolate the influence of one mouse on a social interaction, assays testing social approach use a three-chambered box in which a mouse chooses between interacting with a restrained mouse or an inanimate object (Landauer and Balster, 1982; Pomerantz et al., 1983; Carter et al., 1995; Winslow, 2003). Social approach data is collected by noting how much time the mouse spends in each of the three chambers, and comparing the amount of time the mouse spends in the chamber with the stimulus mouse to the time spent with the inanimate object. These data can be collected in a number of ways: by a trained observer viewing chamber crossings (Brodkin et al., 2004; Brigman et al., 2009), by counting infrared beam breaks between chambers of the apparatus (Nadler et al., 2004), or by using video recording and computer software to track the mouse's movement in the apparatus (Kwon et al., 2006; Tabuchi et al., 2007; Blundell et al., 2009). The ability to track a mouse's movements allows a more sensitive output, as behaviors may be recorded in addition to chamber crossings. Various tools exist to study mouse behavior which can track mouse movement during social approach. Software packages such as Ethovision (Noldus), SMART Triwise Video Tracking (Harvard Apparatus), or VideoMot2 (TSE), have features which allow the user to track movement and specific aspects of mouse behavior such as motion, rearing, head orientation, measure interactions between multiple mice, and some (Ethovision) are able to detect behaviors such as grooming, fighting, or tail rattling. These tools offer many options for analyzing video recorded animal behavior, however, commercially available tools may be prohibitively expensive for individual researchers or small laboratories. A number of free programs exist that may be used for video analysis of social behavior, such as Image) ${ }^{1}$ (Nakajima

'http://rsb.info.nih.gov/ij/ 
et al., 2008; Nakatani et al., 2009; Tanda et al., 2009) and WinTrack ${ }^{2}$. These programs allow users to write custom scripts for specialized applications. It would be advantageous if standardized tests and analysis methods for social interaction phenotypes were available to laboratories carrying out mouse behavioral phenotyping batteries for comprehensive studies of mouse models of human disease (van der Staay and Steckler, 2001; Solberg et al., 2006; Crawley, 2007); such methods must be inexpensive, easy to implement, and allow for reproducibility between users and laboratories.

Here we present a novel, rapid and flexible computer-assisted method for analyzing social approach data collected by video, using scripts written for an image analysis program freely available from the National Institutes of Health (NIH). Using this method, a mouse can be tracked as it moves within chambers, as well as between chambers. We present social approach data on C57BL/6 mice, a frequently studied inbred strain shown to exhibit high levels of sociability in social approach assays (Moy et al., 2007). We show that the computer-assisted automated scoring method is comparable in accuracy to a trained human observer, and present a method of analysis of the social perimeter as a measure of direct social interaction within the chambers.

\section{MATERIALS AND METHODS ANIMALS}

Mice used as test subjects in social approach were experimentally naïve 8 week old male C57BL/6 mice obtained from Taconic (Hudson, NY, USA) at 7 weeks of age. Unrelated age-matched $\mathrm{C} 57 \mathrm{BL} / 6$ male mice unfamiliar to the test mice were used as the social stimulus. All mice were housed five to a cage throughout testing, on a 12-h light/dark cycle, with ad libitum access to food and water. Behavioral testing and handling took place during the light cycle. Mice were handled by an experimenter for approximately $5 \mathrm{~min}$ a day for 2 days prior to testing. On the day of the experiment, mice were moved to the behavior testing room in their home cages and allowed to acclimate for $45 \mathrm{~min}$. Mice to be used as the social stimulus were placed into the plastic holding cages for 5 min to acclimate, then removed. Stimulus mice were used in only one trial on any given testing day. Experiments were performed according to a protocol approved by the Massachusetts Institute of Technology Committee on Animal Care and in accordance with NIH guidelines.

\section{APPARATUS}

The social approach apparatus was built from opaque acrylic sheets (US Plastics, Lima, OH, USA) bonded using acrylic solvent (GE Polymershapes, Huntsville, NC, USA) to form an open top box (Figure 1A). The box measured 24 " $\mathrm{L} \times 12$ " $\mathrm{W} \times 12$ " $\mathrm{H}$, divided into three chambers $(8$ " $\mathrm{L} \times 12$ "W) by acrylic sheets with 3 " $\times 3$ " square openings cut into them to allow test mice to move between chambers. The sides and partitions of the box were made of black acrylic, while the floor was made of white acrylic to create a high contrast between the floor and the dark colored mouse strain used in this study. The apparatus was placed in the behavior testing room in a position where the box was evenly lit at 300 lux, as tested using a light meter (Sekonic). The apparatus chambers were numbered

${ }^{2}$ http://www.dpwolfer.ch/wintrack/index.html
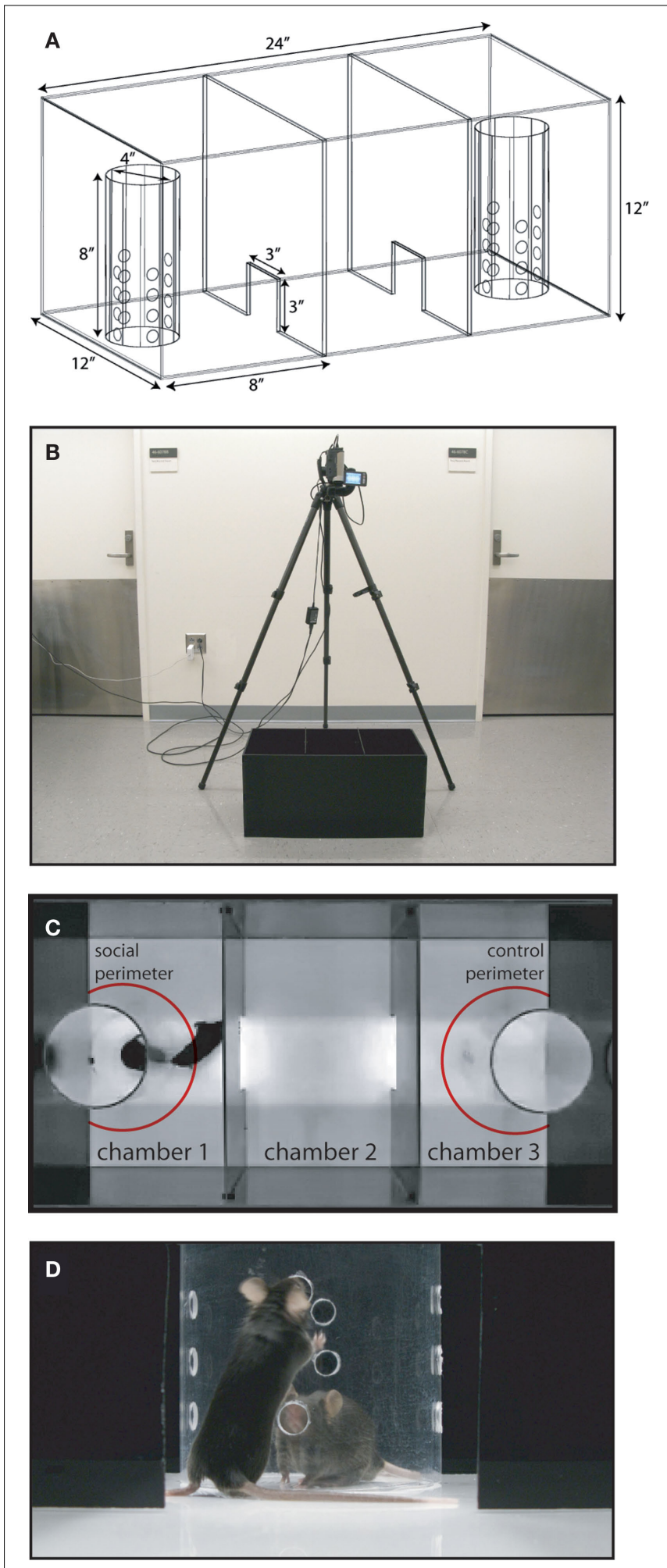

FIGURE 1 | Social approach apparatus setup. (A) Schema of social approach apparatus, showing dimensions. (B) Placement of video camera for recording social approach behavior. (C) Top down view of social approach box, with stimulus mouse in chamber 1 . Subject mouse shown is interacting within the social perimeter, indicated in red. (D) View of subject and stimulus mouse interacting in chamber 1. 
1-3 from left to right for reference. Chambers 1 and 3 contained cages. The cage in chamber 1 contained a social stimulus mouse (Figure 1C); the cage in chamber 3 was left empty as a control. The cages used were clear acrylic cylinders measuring 8 " $\mathrm{H} \times 4$ " $\mathrm{D}$ (US Plastics, Lima, OH, USA) drilled with numerous 1/4" holes to a height of 4 ". The cylinders were large enough for an adult mouse to move about comfortably, and the height and mass of the cylinders prevented animals from climbing into or out of the cylinder, or from displacing the cylinder during acclimation and testing (Figure 1D).

\section{SOCIAL APPROACH}

Immediately prior to testing, each subject mouse was acclimated to the social approach apparatus. An experimenter placed a subject mouse into chamber 2 of the clean empty social approach box, allowing it to explore all three chambers for $5 \mathrm{~min}$. Video recordings taken during this time were analyzed to test whether mice showed chamber preference. At the end of $5 \mathrm{~min}$, the subject mouse was removed, and a previously acclimated stimulus mouse was placed into the empty cage in chamber 1 of the social approach box. The test mouse was replaced in chamber 2 and video recording was taken as the test mouse explored the box and cages for $10 \mathrm{~min}$. The stimulus mouse held in the cage in chamber 1 was available to the subject mouse for visual, tactile, and olfactory contact, but only at the initiation of the subject mouse. The subject mouse's activity was videotaped for analysis of time spent in each chamber, and time spent in the "social" and "control" perimeters. At the end of the 10-min testing period, the subject and stimulus mice were removed and the apparatus and cages were wiped thoroughly with Quatricide PV (Pharmacal, Naugatuck, CT, USA) to remove odors.

\section{ACQUISITION AND PROCESSING OF DATA Video acquisition}

Video recordings of social approach trials were taken with a digital camcorder (Sony Handycam) held directly above the apparatus at a height of 3" using a tripod (Manfrotto) (Figure 1B). The position of the tripod and the social approach box were fixed using markings on the floor of the behavior room, position was checked frequently. Correct calibration of camera zoom is essential for downstream analysis of videos. To do this, the camera zoom was set so that the floor of the social approach apparatus fit precisely into a $120 \times 60$ pixel box in the exported video (see below). Videos were recoded at $320 \times 240$ pixels, 30 frames per second, using Quicktime Pro 7.1.3 software (Apple) run on a PowerBook G4 (Apple) laptop computer with GeForce FX Go5200 graphics card and 512 MB RAM. To reduce file size to approximately $325 \mathrm{~KB}$, images were exported at $160 \times 120$ pixels, 3 frames per second for subsequent analysis, using options in Quicktime's export settings.

\section{Processing of data}

(1) Install Image (from the $\mathrm{NIH}^{3}$, installation instructions are included) to data processing computer. ImageJ is available for Microsoft Windows and Linux operating systems, however, the scripts have only been verified using Mac.

${ }^{3}$ http://rsb.info.nih.gov/ij/
(2) Install custom scripts downloaded from http://www. frontiersin.org/behavioralneuroscience/paper/10.3389/ neuro.08/048.2009/ by moving these seven scripts into the folder Image $\backslash$ plugins $\backslash$ Macros.

(3) Load video files using the File $>$ Open function. At this stage users are given the option of converting video files to grayscale, this is advised.

(4) Run scripts in order to analyze video (Figure 2). Scripts can be accessed from the Plugins $>$ Macros dropdown menu.

- The "Step 1 field selection" script file defines a $120 \times 60$ pixel box. Place box to precisely fit the floor of the social approach apparatus. If the video camera or box position were to accidentally shift during the course of an experiment such that floor no longer precisely fits the $120 \times 60$ pixel box, the movie file can be adjusted post-recording using Image $>$ Adjust $>$ Size (to correct a problem with zoom) or Image $>$ Rotate $>$ Arbitrarily (to correct a problem with rotation).

- The "Step 2 blocking" script blocks out the area of the cages and partitions of the apparatus to remove signal from these areas in downstream analysis.

- The “Step 3 chamber 1 ROI” script uses ImageJ's thresholding, noise reduction, and variance filters (more information about these can be found at http://rsb.info.nih. gov/ij/docs/menus/image.html) to generate a signal from the mouse that can be analyzed against a background with minimal noise. The output from the "chamber 1 ROI" script is a $Z$-axis plot of mean gray value signal within the region of interest corresponding to chamber 1 for the recording time. Copy and paste $Z$-axis plot to data sheet using the "copy" function.

- The "Step 4 chamber 2 ROI" obtains the same information from chamber 2, $Z$-axis plot should be transferred to data sheet.

- The "Step 5 chamber 3 ROI" obtains the same information from chamber 3, $Z$-axis plot should be transferred to data sheet.

- The "Step 6 social perimeter" and "Step 7 control perimeter" scripts generate $Z$-axis grey value plots for regions of interest corresponding to a perimeter centered on the cage in chamber 1 (social perimeter) or chamber 3 (control perimeter), which is 1.5 times larger than the circumference of the cage. This allows for quantifying the time spent in close proximity to the cage, where social interaction is likely to take place, as opposed to time spent in the chamber but not in close proximity to the cage. Transfer data for each to data sheet.

(5) Review data in data sheet to check for gaps and conflicts as described below. After checking for errors, calculate percent time spent in each chamber in Excel. We do this by dividing the number of frames containing signal for a given ROI by the total number of frames for the assay (this should be 1800 frames for a 10-min movie exported at 3 frames per second) and multiplying by 100 .

\section{Verification of data and analysis}

The "chamber 1 ROI", "chamber 2 ROI" and "chamber 3 ROI" scripts are designed so that the output of these minimizes the occurrence of gaps (resulting from no mouse signal in any chamber 


\begin{tabular}{|c|c|}
\hline step & macro script \\
\hline field selection & $\begin{array}{l}\text { makeRectangle }(0,0,120,60) ; \\
\text { // Hand center rectangle in center } \\
\text { // of social approach box floor. } \\
\text { // Check camera zoom to verify that } \\
\text { // rectangle fits social approach box } \\
/ / \text { floor. }\end{array}$ \\
\hline blocking & 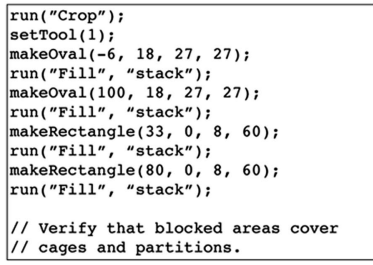 \\
\hline
\end{tabular}

chamber 1 ROI

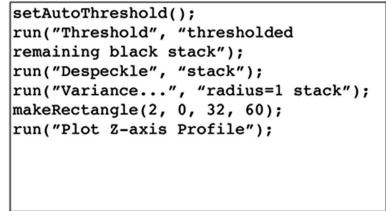

chamber 2 ROI

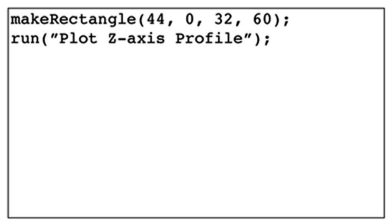

chamber 3 ROI

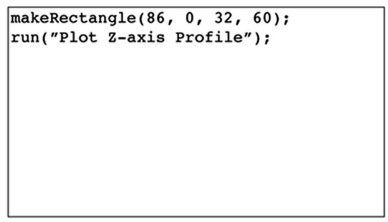

social perimeter

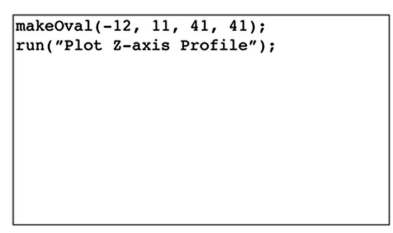

control perimeter

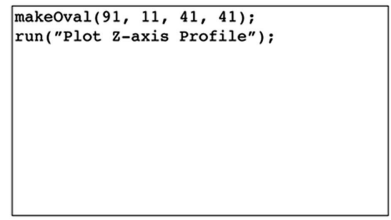

movie file
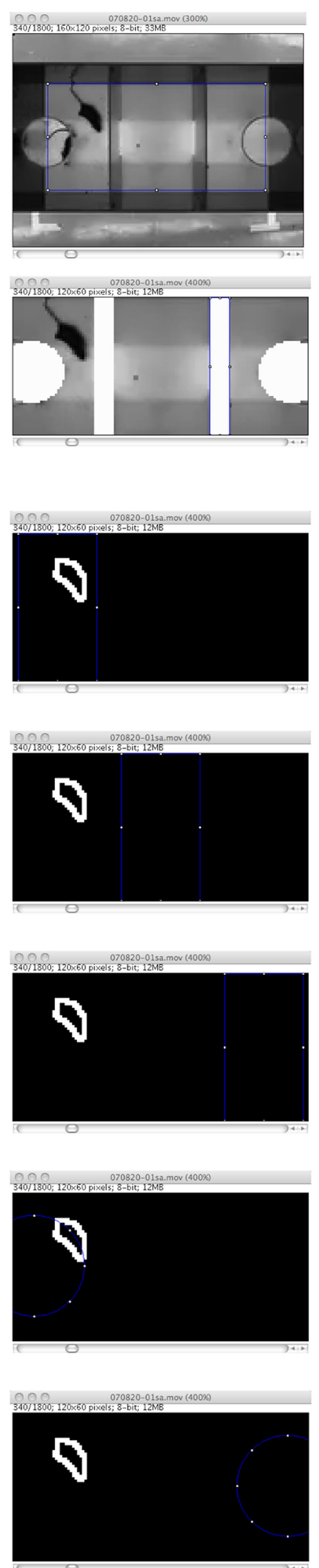

\section{data output}

no output

no output

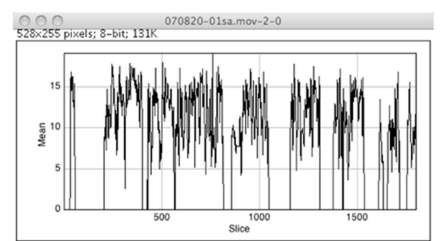

(List) Save... Copy...

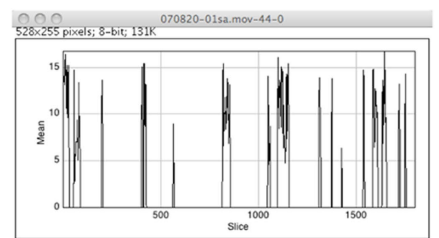

(List) Save... Copp... $x=1785, y=0$

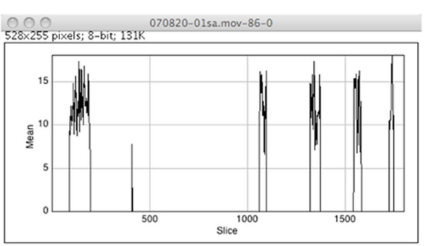

(List) (Save... Copy... $x=677, y=0$

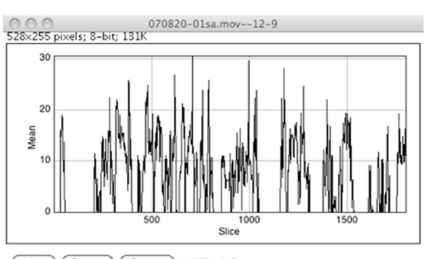

$$
\text { List) (Save... Copy... } x=157, y=0
$$

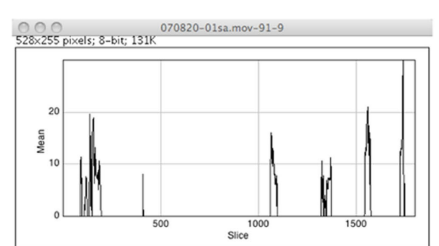

(List) (Save...) Coopy... $x=233, \mathrm{y}=0$

FIGURE 2 | Computer-assisted scoring of social approach. Processing of social approach movie files using ImageJ computer scripts. Scripts are run in order shown, data output shows signal in $\mathrm{ROI}$ over each frame of video. 
region of interest in a frame, a "disappearing mouse") and conflicts (resulting from signal from the mouse occurring in two chamber regions of interest in the same frame, "two mice"). However, both gaps and conflicts occur at a low rate and thus the data for all regions of interest should be hand-checked for accuracy by reviewing raw data for errors, and verified by comparing to the original video. If a gap is present, caused by the signal for the mouse briefly dropping below threshold, a notation is made to count signal in the gap areas. If a conflict is present, caused by signal for the mouse appearing in ROIs for two chambers at the same time (e.g. as the mouse moved between chambers), a chamber entry is recorded, and signal from the exiting chamber not counted, if the mouse's head and shoulders move into the chamber. After hand-checking the data, the number of frames containing mouse signal is summed for each chamber and used to calculate percent time spent in each chamber over the course of the assay. The percent time spent in the social versus control perimeter is calculated by summing the number of frames containing mouse signal and dividing this by the total number of frames for the video.

\section{Statistics}

Analysis of social approach behavior was carried out using oneway ANOVA, comparing the number of frames containing mouse signal between ROIs. Where a significant effect was seen, post hoc comparison was performed using Tukey HSD test. The significance level was set at $P<0.05$. Comparison between hand and computer-assisted scored social approach data was carried out using two-way ANOVA.

\section{RESULTS}

To test whether mice run in the social approach apparatus showed a chamber preference in the absence of a stimulus mouse, we analyzed acclimation trials for time spent in chambers 1, 2, and 3. In all acclimation trials, mice spent similar amounts of time exploring chambers 1 and 3 (Figure 3A), giving evidence that the mice do not exhibit chamber preference when a stimulus mouse is not present. It would be expected that an inconsistency in light, noise, or odors across the three chambers would be reflected in chamber preference during acclimation. An observed side preference that persisted after known variables in the apparatus were controlled for would limit confidence in any social approach data resulting from testing. In such a situation, the apparatus should be relocated to an area where no side preference is seen during acclimation.

During the social approach trials, the addition of a stimulus mouse to chamber 1 caused subject mice to significantly alter their behavior, spending the majority of the assay exploring the chamber containing the stimulus mouse (Figure 3B). The change was consistent, with 11 of the 12 mice tested showing a preference for the stimulus mouse in chamber 1 . To test the time course of investigation over the 10-min assay, social approach data were separated into four $150 \mathrm{~s}$ bins, and time spent in chamber 1 for each bin was measured. It was found that mice exhibited the most social approach behavior during the first $150 \mathrm{~s}$ bin, with time spent in chamber 1 dropping off as the assay progressed (Figure 3C), similar to what was found in a previous study (Nadler et al., 2004). As a test to determine how much of the subject mouse's time in chamber 1 or 3 was spent investigating the cages, a measure of time spent within
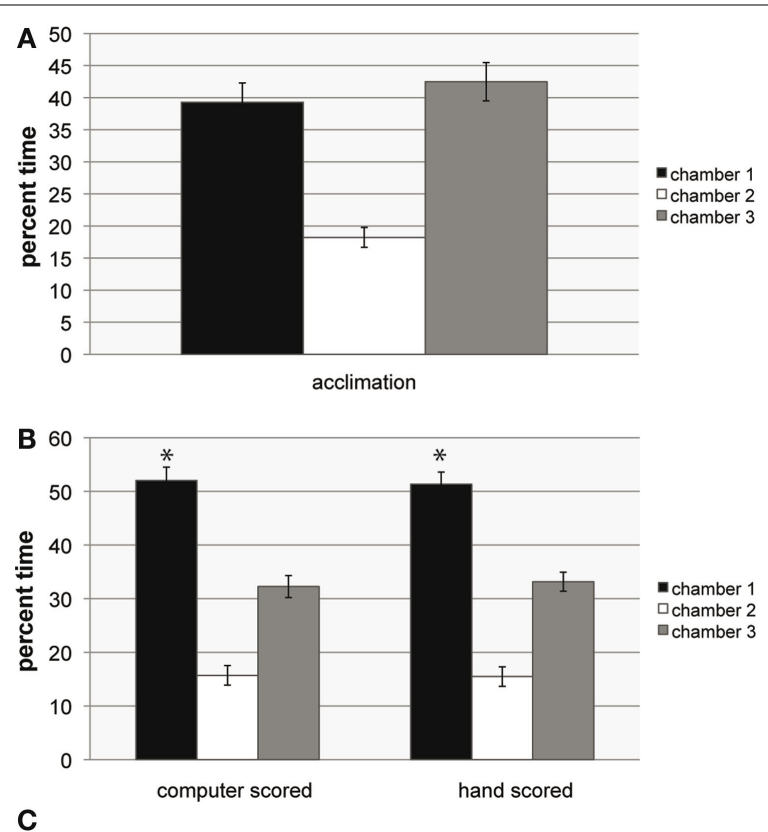

C
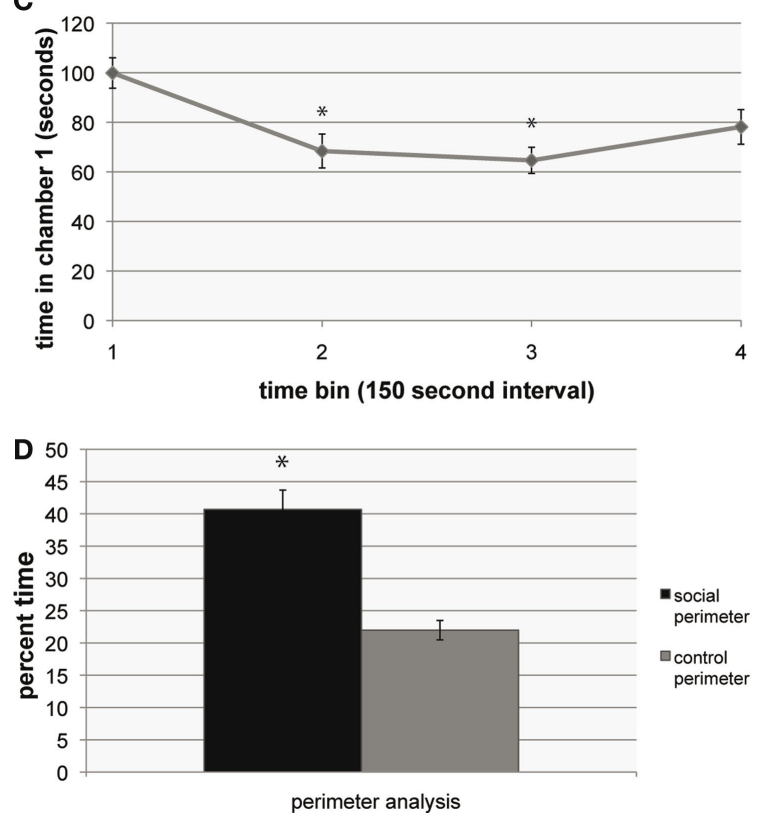

FIGURE 3 | Social approach data. (A) Data showing social approach behavior. All trials use male C57BL/6 mice, tested at 8 weeks of age. For (A-C), $n=12$, for (D), $n=10$. Error bars indicate SEM. (A) Percent time spent in chambers 1, 2, and 3 during 5-min acclimation. (B) Comparison of computer-assisted scoring and experimenter scoring of 10-min social approach trials with stimulus mouse in chamber 1 . Significant preference was found for percent time spent in chamber 1 compared to chamber $3,{ }^{*} P<0.05$, ANOVA within group comparison between chamber 1 and 3 . No significant difference was found between computer scored and experimenter scored data. (C) Time spent in chamber 1 by the subject mouse over the course of the 10-min trial, based on computer scored data. The trial was divided into four bins of equal length (150 s). The amount of time spent in chamber 1 in bins 2 and 3 was significantly less than in bin 1, based on ANOVA comparison between bins, ${ }^{*} P<0.01$ (Tukey HSD test). (D) Percent time spent in social perimeter (within chamber 1) compared to control perimeter (within chamber 3). Significant preference for social perimeter, ${ }^{*} P<0.05$, ANOVA within group comparison between social and control perimeter. 
a circumference 1.5 times that of the cage was taken for chamber 1 ("social perimeter") and chamber 3 ("control perimeter"). For both chamber 1 and chamber 3, the majority of time in each chamber was spent in proximity to the cage (Figure 3D). These data are consistent with other reports that have used similar measurements of proximity of the subject mouse to the stimulus mouse (Kwon et al., 2006; Tabuchi et al., 2007; Nakajima et al., 2008; Nakatani et al., 2009; Tanda et al., 2009).

To verify the accuracy of the computer scripts in measuring the time each mouse spent in the chambers, all movies were also hand scored by trained researchers. Researchers watched the movie file and recorded the time at which each chamber crossing took place, and summed the time spent in each of the chambers. Within each scoring group, there was a significant preference for chamber 1 $\left(F_{(2,71)}=154.8, P<0.001\right)$. Importantly, there were no significant effect of hand scoring versus computer-assisted scoring of data $\left(F_{(1,71)}=0, P=1\right)$ (Figure 3B).

\section{DISCUSSION}

The computer-assisted scoring method for social approach offers a standardized way to study social interaction in mouse models of human disease in a controlled environment while maintaining the sensitivity to measure the test mouse's movements within the chamber. A concern with any behavioral phenotyping test is reproducibility across laboratories (Crabbe et al., 1999). However, social approach assays appear to be reproducible among inbred mouse strains across laboratories. To show accuracy and reproducibility of the social approach assay in general and the computer-assisted method in particular, we first compared our computer-assisted social approach data with hand scored data, and found no difference between the two methods. When our results are compared to those of other studies examining social approach in C57BL/6 mice using three-chambered apparatuses of varying dimensions (Crawley et al., 2007; Labrie et al., 2008; McFarlane et al., 2008), there was no substantial difference in chamber preference, even in cases where the mice tested were 5-6 weeks of age (Moy et al., 2004), or female (Brodkin et al., 2004). Mice that had been subjected to prior behavioral phenotyping such as open field anxiety and rotarod muscle coordination tests showed no difference in social approach preference when compared to the mice tested in this study (Moy et al., 2007), nor were variations noted when a mouse of a different strain, such as DBA/2J or A/J, was used as a stimulus (Brodkin et al., 2004; Nadler et al., 2004).

\section{REFERENCES}

Blanchard, D. C., Griebel, G., and Blanchard, R. J. (2001). Mouse defensive behaviors: pharmacological and behavioral assays for anxiety and panic. Neurosci. Biobehav. Rev. 25, 205-218.

Blanchard, R. J., and Blanchard, D. C. (2003). Bringing natural behaviors into the laboratory: a tribute to Paul MacLean. Physiol. Behav. 79, 515-524.

Blundell, J., Tabuchi, K., Bolliger, M. F., Blaiss, C. A., Brose, N., Liu, X.,

It is important to note that other inbred strains tested in social approach assays often exhibit different phenotypes: FVB and DBA/2 mice show preference for chamber 1 similar to that exhibited by C57BL/6 mice, while BALB, BTBR, and 129S1 mice tend to be less sociable (Moy et al., 2007), so caution must be taken to consider background strain when testing or comparing social approach behaviors in mouse models of disease. This assay can also be used to screen for genetic modifiers of social behavior. As an example, we have tested mice double haploinsufficient for the candidate autism susceptibility genes Pten and Slc6a4 in an isogenic C57BL/6 background, and found evidence that these genes interact to influence social approach behavior (Page et al., 2009).

While the tools presented here do not have the sensitivity to measure detailed aspects of social behavior, such as time engaging in active sniffing, or aggressive behaviors such as tail rattling, the analysis of social perimeter offers a measurement of the time in which specific social investigation is most likely to occur. The computerassisted method of analyzing social behavior data is readily adaptable. By altering the computer scripts used for analysis, it becomes possible to extract additional data on the test mouse without carrying out additional assays. For example, locomotion and distance covered in the social approach apparatus can be measured during social approach or acclimation, and used to examine differences in locomotor activity between genotypes being tested. This computerassisted three-chambered social approach paradigm may be repeated after a period of separation of subject and stimulus mouse to measure social recognition (Page et al., 2009), and variations could be made to the apparatus, such as the use of a red floor to create a contrasting background for mouse strains with varying coat colors. We have used altered versions of the scripts to analyze data gathered on different types of behavioral apparatuses, for example measuring thigmotaxis in a test of open field anxiety and crossings over a false precipice in a visual cliff test of vision (data not shown) and expect that users of this method will modify the scripts presented here to optimize for their particular needs. Using computer-assisted scoring, previously observation intensive behavioral assays can be run in a reproducible way by laboratories, without the need for expensive proprietary software or a large team of trained observers.

\section{ACKNOWLEDGMENTS}

This work was supported by funding from the Nancy Lurie Marks Family Foundation (DP), the Simons Foundation and the Autism Consortium. We thank Chrysa Prestia for helpful discussions.

approach-avoidance behavior of inbred mouse strains towards DBA/2 mice. Brain Res. 1002, 151-157.

Carter, C. S., DeVries, A. C., and Getz, L. L. (1995). Physiological substrates of mammalian monogamy: the prairie vole model. Neurosci. Biobehav. Rev. 19, 303-314.

Crabbe, J. C., Wahlsten, D., and Dudek, B. C. (1999). Genetics of mouse behavior: interactions with laboratory environment. Science 284, 1670-1672.

Crawley, J. N. (2004). Designing mouse behavioral tasks relevant to autistic-like behaviors. Ment. Retard Dev. Disabil. Res. Rev. 10, 248-258.

Crawley, J. N. (2007). Mouse behavioral assays relevant to the symptoms of autism. Brain Pathol. 17, 448-459.

Crawley, J. N., Chen, T., Puri, A., Washburn, R., Sullivan, T.L.,Hill, J. M., Young, N. B., Nadler, J. J., Moy, S. S., Young, L. J., Caldwell, H. K., and Young, W. S. (2007). Social approach behaviors in oxytocin knockout mice: comparison of two independent lines tested in different laboratory environments. Neuropeptides 41, 145-163. 
Cutler, M. G. (1991). An ethological study of the effects of buspirone and the 5-HT3 receptor antagonist, BRL 43694 (granisetron) on behaviour during social interactions in female and male mice. Neuropharmacology 30, 299-306.

Ferguson, J. N., Aldag, J. M., Insel, T. R., and Young, L. J. (2001). Oxytocin in the medial amygdala is essential for social recognition in the mouse. J. Neurosci. 21, 8278-8285.

Griebel, G., Belzung, C., Perrault, G., and Sanger, D. J. (2000). Differences in anxiety-related behaviours and in sensitivity to diazepam in inbred and outbred strains of mice. Psychopharmacology (Berl.) 148, 164-170.

Kwon, C. H., Luikart, B. W., Powell, C. M., Zhou, J., Matheny, S. A., Zhang, W., Li, Y., Baker, S. J., and Parada, L. F. (2006). Pten regulates neuronal arborization and social interaction in mice. Neuron 50, 377-388.

Labrie, V., Lipina, T., and Roder, J. C. (2008). Mice with reduced NMDA receptor glycine affinity model some of the negative and cognitive symptoms of schizophrenia. Psychopharmacology (Berl.) 200, 217-230.

Landauer, M. R., and Balster, R. L. (1982). A new test for social investigation in mice: effects of d-amphetamine. Psychopharmacology (Berl.) 78, 322-325.

Laviola, G., Terranova, M. L., Sedowofia, K., Clayton, R., and Manning, A. (1994). A mouse model of early social interactions after prenatal drug exposure: a genetic investigation. Psychopharmacology (Berl.) 113, 388-394.

Long, J. M., LaPorte, P., Paylor, R., and Wynshaw-Boris, A. (2004). Expanded characterization of the social interaction abnormalities in mice lacking Dvll. Genes Brain Behav. 3, 51-62.

McFarlane, H. G., Kusek, G. K., Yang, M., Phoenix, J. L., Bolivar, V. J., and Crawley, J. N. (2008). Autism-like behavioral phenotypes in BTBR T+tf/J mice. Genes Brain Behav. 7, 152-163.

Mondragon, R., Mayagoitia, L., LopezLujan, A., and Diaz, J. L. (1987). Social structure features in three inbred strains of mice, $\mathrm{C} 57 \mathrm{Bl} / 6 \mathrm{~J}, \mathrm{Balb} / \mathrm{cj}$, and NIH: a comparative study. Behav. Neural Biol. 47, 384-391.

Moretti, P., Bouwknecht, J. A., Teague, R., Paylor, R., and Zoghbi, H. Y. (2005). Abnormalities of social interactions and home-cage behavior in a mouse model of Rett syndrome. Hum. Mol. Genet. 14, 205-220.

Moy, S. S., Nadler, J.J., Perez, A., Barbaro, R. P., Johns, J. M., Magnuson, T. R., Piven, J., and Crawley, J.N. (2004). Sociability and preference for social novelty in five inbred strains: an approach to assess autistic-like behavior in mice. Genes Brain Behav. 3, 287-302.

Moy,S.S.,Nadler,J.J.,Young, N.B.,Perez,A., Holloway, L. P., Barbaro, R. P., Barbaro, J. R., Wilson, L. M., Threadgill, D. W., Lauder, J. M., Magnuson, T. R., and Crawley, J. N. (2007). Mouse behavioral tasks relevant to autism: phenotypes of 10 inbred strains. Behav. Brain Res. 176, 4-20.

Nadler, J. J., Moy, S. S., Dold, G., Trang, D., Simmons, N., Perez, A., Young, N. B., Barbaro, R. P., Piven, J., Magnuson, T. R., and Crawley, J. N. (2004). Automated apparatus for quantitation of social approach behaviors in mice. Genes Brain Behav. 3, 303-314.

Nakajima, R., Takao, K., Huang, S. M., Takano, J., Iwata, N., Miyakawa, T., and Saido, T. C. (2008). Comprehensive behavioral phenotyping of calpastatinknockout mice. Mol Brain 1, 7 .

Nakatani, J., Tamada, K., Hatanaka, F., Ise, S., Ohta, H., Inoue, K., Tomonaga, S., Watanabe, Y., Chung, Y. J., Banerjee, R., Iwamoto, K., Kato, T., Okazawa, M., Yamauchi, K., Tanda, K., Takao, K., Miyakawa, T., Bradley, A., and Takumi, T. (2009). Abnormal behavior in a chromosome-engineered mouse model for human 15q11-13 duplication seen in autism. Cell 137, 1235-1246.

O'Tuathaigh, C. M., Babovic, D., O'Sullivan, G. J., Clifford, J. J., Tighe, O., Croke, D. T., Harvey, R., and Waddington, J. L. (2007). Phenotypic characterization of spatial cognition and social behavior in mice with 'knockout' of the schizophrenia risk gene neuregulin 1. Neuroscience 147 , 18-27.

Page, D. T., Kuti, O. J., Prestia, C., and Sur M. (2009). Haploinsufficiency for Pten and Serotonin transporter cooperatively influences brain size and social behavior. Proc. Natl. Acad. Sci. U.S.A. 106, 1989-1994.

Pomerantz, S. M., Nunez, A. A., and Bean, N. J. (1983). Female behavior is affected by male ultrasonic vocalizations in house mice. Physiol. Behav. 31, 91-96.

Rodriguiz, R. M., Chu, R., Caron, M. G., and Wetsel, W. C. (2004). Aberrant responses in social interaction of dopamine transporter knockout mice. Behav. Brain Res. 148, 185-198.

Shi, L., Fatemi, S. H., Sidwell, R. W., and Patterson, P. H. (2003). Maternal influenza infection causes marked behavioral and pharmacological changes in the offspring. J. Neurosci. 23, 297-302.

Solberg, L. C., Valdar, W., Gauguier, D., Nunez, G., Taylor, A., Burnett, S., Arboledas-Hita, C., HernandezPliego, P., Davidson, S., Burns, P., Bhattacharya, S., Hough, T., Higgs, D., Klenerman, P., Cookson, W.O., Zhang, Y., Deacon, R. M., Rawlins, J. N., Mott, R., and Flint, J. (2006). A protocol for high-throughput phenotyping, suitable for quantitative trait analysis in mice. Mamm. Genome 17, 129-146.

Tabuchi, K., Blundell, J., Etherton, M. R., Hammer, R. E., Liu, X., Powell, C. M., and Sudhof, T.C.(2007).A neuroligin-3 mutation implicated in autism increases inhibitory synaptic transmission in mice. Science 318, 71-76.

Takao, K., Yamasaki, N., and Miyakawa, T. (2007). Impact of brain-behavior phenotypying of genetically-engineered mice on research of neuropsychiatric disorders. Neurosci. Res. 58, 124-132.

Tanda, K., Nishi, A., Matsuo, N., Nakanishi, K., Yamasaki, N., Sugimoto, T., Toyama, K., Takao, K., and Miyakawa, T. (2009). Abnormal social behavior, hyperactivity, impaired remote spatial memory, and increased D1-mediated dopaminergic signaling in neuronal nitric oxide synthase knockout mice. Mol Brain 2, 19.

Tecott, L. H. (2003). The genes and brains of mice and men.Am. J. Psychiatry 160 , 646-656.

van der Staay, F. J., and Steckler, T. (2001). Behavioural phenotyping of mouse mutants. Behav. Brain Res. 125, 3-12.

Wersinger, S. R., Caldwell, H. K., Christiansen, M., and Young, W. S. 3rd (2007). Disruption of the vasopressin $1 \mathrm{~b}$ receptor gene impairs the attack component of aggressive behavior in mice. Genes Brain Behav. 6, 653-660.

Wills, G. D., Wesley, A. L., Moore, F. R., and Sisemore, D. A. (1983). Social interactions among rodent conspecifics: a review of experimental paradigms. Neurosci. Biobehav. Rev. 7,315-323.

Winslow, J. T. (2003). Mouse Social Recognition and Preference. Current Protocols in Neuroscience/Editorial Board, Chapter 8, Unit 8. Somerset, NJ, John Wiley \& Sons, pp. 16.

Winslow, J. T., and Camacho, F. (1995). Cholinergic modulation of a decrement in social investigation following repeated contacts between mice. Psychopharmacology 121, 164-172.

Conflict of Interest Statement: The authors declare that the research was conducted in the absence of any commercial or financial relationships that could be construed as a potential conflict of interest.

Received: 01 August 2009; paper pending published: 24 August 2009; accepted: 29 October 2009; published online: 30 November 2009.

Citation:Page DT,KutiOJand SurM (2009) Computerized assessment of social approach behavior in mouse. Front. Behav. Neurosci. 3:48. doi: 10.3389/neuro.08.048.2009 Copyright $\odot 2009$ Page, Kuti and Sur. This is an open-access article subject to an exclusive license agreement between the authors and the Frontiers Research Foundation, which permits unrestricted use, distribution, and reproduction in any medium, provided the original authors and source are credited. 